\title{
Human Embryonic Stem Cells in the Treatment of Patients With Down Syndrome: A Case Report
}

\author{
Geeta Shroff
}

\begin{abstract}
Down syndrome (DS) is a common chromosomal disorder caused by trisomy of chromosome 21 (HSA21q). Individuals with DS suffer from various congenital and progressive diseases. There is no standard treatment for DS. Due to overexpression of genes in trisomic cells, abnormal neuronal development and functional changes occur in the central nervous system of DS patients. The key deficiencies in neural stem and progenitor cell expansion lead to cognitive disabilities in DS. Human embryonic stem cells (hESCs) could serve as an expandable source for neurons production, which could be applied for the treatment of various diseases affecting brain. In this article, we report a case of DS patient treated with hESC therapy. A DS child had delayed milestones, with no speech, subnormal understanding and subnormal motor skills. Following the treatment, patient showed remarkable changes and improvement in the clinical conditions such as better understanding, improved muscle tone of limbs and ability to recognize near ones. hESC therapy was found to be beneficial in the treatment of DS. Further studies are needed to understand the clinical utility of hESC therapy in the patients with DS.
\end{abstract}

Keywords: Down syndrome; Human embryonic stem cells; Trisomy; Alzheimer's disease; Central nervous system

\section{Introduction}

Down syndrome (DS) is a common chromosomal disorder caused by trisomy of chromosome 21 (HSA21q). Individuals with DS suffer from several congenital and progressive medical conditions such as mental retardation, congenital heart disease, gastrointestinal anomalies, skeletal anomalies, leukemia and Alzheimer's disease (AD). Worldwide, the prevalence of DS varies from $1 / 1,000$ to $1 / 1,100$ live births $[1,2]$.

DS is the most frequent genetic cause of intellectual dis-

Manuscript accepted for publication February 24, 2016

Department of Stem Cell Therapy, Nutech Mediworld, H-8, Green Park Extension, New Delhi 110016, India. Email: geetashroff@hotmail.com

doi: http://dx.doi.org/10.14740/jmc2455w ability (ID). Individuals with DS have mean intelligence quotients of about 50 [3]. There is no standard treatment for DS. However, physical therapy, speech-language therapy, occupational therapy, emotional and behavioral therapies, amino acid supplements and other drugs may be used to reduce the symptoms of DS [4].

An extra copy of chromosome 21 causes persistent developmental delays. Abnormal development and functional changes in the neurons cells due to overexpression of genes in trisomic cells lead to abnormalities in the central nervous system (CNS) of DS patients. DS mouse models demonstrate key deficiencies in the neural stem cell (NSC) and progenitor cell expansion resulting in cognitive disabilities in DS [2]. DS-induced pluripotent stem cells (DS-iPSCs) are differentiated into neuro-epithelial progenitor cells (NPCs) and mature forebrain neurons, recapitulating the developmental process of the disease [5]. Human embryonic stem cells (hESCs) have regenerative potential that allows the damaged part of the brain to be repaired. Recently, we have observed that hESCs have the ability to differentiate into different types of neuronal cells. NPCs from hESCs could serve as an expandable source for neurons production, which could be applied for the treatment of various diseases affecting brain.

In our previous studies, we have used hESCs to treat various incurable conditions such as spinal cord injury, cerebral palsy, cortical visual impairment, spinocerebellar ataxia, and Friedreich's ataxia [6-8]. In this article, we report a case of DS patient treated with hESC therapy.

\section{Methodology}

The cells used in this study were cultured and maintained as per our in-house patented technology (patent WO 2007/141657A PCT/1B 2007, published on December 13, 2007) in a good manufacturing practice (GMP), good laboratory practice (GLP) and good tissue practice (GTP) certified laboratory at Nutech Mediworld [9].

The parents provided written informed consent prior to the start of the treatment session. An independent institutional ethics committee (IEC) of Nutech Mediworld approved the study. The evidence for the use of hESCs at our facility has been submitted and accepted at House of Lords, Regenerative Medicine, Science and Technology Committee [9]. 
hESCs therapy is a clinically tested cell-based therapy for neurological disorders. The establishment and characterization of a neuronal cell line derived from human embryo without providing any culture feeder layer embryo has been elaborated elsewhere [6]. The safety of hESCs in the treatment of various terminal or incurable diseases has also been established [10].

The hESCs administered comprised neuronal stem cell progenitors or hematopoietic stem cell progenitors alone or in combination. The routes of administration include intravenous injection $(0.25 \mathrm{~mL}$ or 750,000 - 1.5 million hESCs $)$, subcutaneous injection $(0.25 \mathrm{~mL}$ or $750,000-1.5$ million hESCs $)$, and intramuscular injection $(0.25 \mathrm{~mL}$ or $750,000-1.5$ million hESCs).

\section{Case Report}

A 2-month-old male child was admitted at our facility on September 01, 2014 with chief complaints of delayed milestones, lack of speech, subnormal understanding and subnormal motor skills.

The patient was a known case of DS, diagnosed at birth. Patient was born preterm due to premature rupture of membranes (PROM) and was kept in intensive care unit (ICU) for 2 days due to respiratory distress. The karyotype of patient was $47, \mathrm{XY},+21$. The cardiac atrial septal defects (ASDs) were also observed in patient with an aortic rim of $2.1 \mathrm{~mm}$. At 2 months of age, the child had slanted eyes, simple palmer crease with no neck holding capacity. The patient's first visit to our hospital was at the age of 2 months.

At our facility, hESCs were given as a primary therapy. The patient underwent two sessions of hESC therapy. The first treatment phase lasted till November 27, 2014. The second course of therapy was started on March 30, 2015. There was an improvement in the patient's condition. The understanding of patient was improved and muscle tone was better in all limbs. He had started babbling and crawling. He was social and was able to recognize his near ones.

After 8 months of the treatment, the child was coherent, babbling, and crawling, and eating ability was better. He was able to stand and walk with support. The palmer crease was normal. Only the pincer grasp was not at par with the age group. No adverse events (AEs) were reported. The patient was discharged on April 11, 2015.

\section{Discussion}

This study presents the clinical outcome of a DS patient treated with hESC therapy. hESCs have not been used previously in clinics due to difficulty in harvesting the cells in a xeno-free environment and fear about AEs such as teratoma formation and immune reactions. In the present case, hESCs were transplanted into the patient for the treatment of DS. We have used in-house cultured hESCs for our patient. The cell line was obtained from a single, spare, expendable fertilized ovum, 24 - $48 \mathrm{~h}$ after fertilization. These cells were cultured and maintained in a xeno-free environment [6]. After the treatment, an improvement was observed in the condition of the patient such as better understanding, better muscle tone of limbs and ability to recognize nearby placed objects.

Patients with DS exhibit an AD-like histopathology in the brain, including amyloid plaque accumulation due to abnormal splicing of amyloid precursor protein (APP) and neurofibrillary tangles mainly generated by hyperphosphorylated tau protein [6]. Cell-based therapies have recently been sought for the treatment of DS. In a study by Kern et al, NSCs were used in the treatment of DS in Ts65Dn trisomic mice. Several clustered granules which contain tau/relin were found in multiple areas of the brain of several mice with this trisomy. After stem cell transplantation, remarkable decrease in these granules was observed. NSCs have unique ability to migrate and express growth factors that might contribute to reducing tau/relin accumulation in aging DS [7].

Recently, it has been reported that the abnormal functioning of the astroglial cells plays a key role in DS. Researchers developed a new cellular model for studying DS. The skin cells of patients with DS were induced into a pluripotent state. PSCs were allowed to develop into neurons and astroglial cells. Astroglial cells act as "helper" cells that assist in the neurons development. It was found that the astroglial cells produced a certain protein in large amounts than normal that could have a negative effect on the development of neurons [11]. DS astroglial cells exhibit higher levels of reactive oxygen species (ROS) and lower levels of synaptogenic molecules and cause abnormal gene expression profiles. This causes toxicity to neurons, and restricts neurogenesis [11]

Stem cells have the potential to communicate with other body cells due to various factors such as chemokines, cytokines and other growth factors released from the site of injury. The cell adhesion molecules (CAMs) such as selectins and integrins are present on the surface of stem cells, which upgrade the whole mechanism [12-14]. In a study by Hibaoui et al, iPSCs were isolated from monozygotic twins with trisomy and injected intramuscularly in animal model with DS. After intramuscular injection, these iPSCs were differentiated into all three embryonic germ layers in vitro. The iPSCs were differentiated into NPCs and further matured into neurons [15]. Human iPSCs share similar features of ESCs. hESCs have the ability to differentiate into several neural cells and tissue. In our previous study, we have shown the mechanism of division and differentiation of single NPC into neurons, axons and neuronal tissue. Thus, we could assume that in our case hESCs might have induced normal neurogenesis in the brain, correcting the abnormal functioning of astroglial cells, thereby, improving the overall condition the DS patient.

Further, silencing extra chromosome in DS could lead to the development of new treatment strategies for this disorder. The RNA gene XIST present on X chromosome is normally responsible for turning off one of the two $\mathrm{X}$ chromosomes found in women. XIST produces a large piece of RNA that coats X chromosomes and condenses it into an inaccessible bundle. XIST can be used to shut down chromosome 21 in trisomic cells. It has been shown that extra copy of 21 chromosome can be silenced in the laboratory using patient-derived stem cells [16]. Researchers have observed that XIST accumulates around every $\mathrm{X}$ chromosome in very young human embryos of 
both male and female [17]. Thus, it could be hypothesized that in our case hESCs might have activated XIST gene, helping in silencing the extra 21 chromosome.

This is the first study to report the use of hESC therapy in a patient with DS. Though beneficial, this investigation needs further research in order to understand the clinical utility of hESC therapy in patients with DS.

\section{Acknowledgement}

The author acknowledges all the doctors, staff and patients of the Nutech Mediworld. The author also acknowledges Knowledge Isotopes Pvt. Ltd for the writing support.

\section{Grant Support}

None.

\section{Conflict of Interest}

The author declares that she has no competing interests.

\section{References}

1. WHO, Genes and human disease, Genes and chromosomal diseases, Down Syndrome. Available from: http:// wwwwhoint/genomics/public/geneticdiseases/en/index$1 \mathrm{html}$.

2. Haydar TF, Reeves RH. Trisomy 21 and early brain development. Trends Neurosci. 2012;35(2):81-91.

3. Chapman RS, Hesketh LJ. Behavioral phenotype of individuals with Down syndrome. Ment Retard Dev Disabil Res Rev. 2000;6(2):84-95.

4. National Institute of Neurological Disorder \& Research. Multiple Sclerosis: Hope Through Research. Available at; http:/www.ninds.nih.gov/disorders/multiple_sclerosis/detail_multiple_sclerosis.htm; Accessed on 19 July, 2015.

5. Chang CY, Chen SM, Lu HE, Lai SM, Lai PS, Shen PW, Chen PY, et al. N-butylidenephthalide attenuates Alzheimer's disease-like cytopathy in Down syndrome induced pluripotent stem cell-derived neurons. Sci Rep.
2015;5:8744.

6. Shroff Geeta. Establishment and Characterization of a Neuronal Cell line derived from a 2-cell Stage Human Embryo: Clinically Tested Cell-based Therapy for Neurological Disorders. International Journal of Recent Scientific Research. 2015;(6):3730-3738.

7. Shroff G, Gupta A, Barthakur JK. Therapeutic potential of human embryonic stem cell transplantation in patients with cerebral palsy. J Transl Med. 2014;12:318.

8. Shroff Geeta, Das L. Human Embryonic Stem Cell Therapy in Cerebral Palsy Children with Cortical Visual Impairment: A Case Series of 40 Patients. J Cell Sci Ther. 2014;5(6): 1 .

9. House of Lords SATSC. Available from http://wwwparliamentuk/documents/lords-committees/science-technology/RegenerativeMedicine/RegenMedpdf; accessed on 26 June 2015.

10. Shroff G, Barthakur JK. Safety of human embryonic stem cells in patients with terminal/incurable conditions- a retrospective analysis. Ann Neurosci. 2015;22(3):132-138.

11. Chen C, Jiang P, Xue H, Peterson SE, Tran HT, McCann AE, Parast MM, et al. Role of astroglia in Down's syndrome revealed by patient-derived human-induced pluripotent stem cells. Nat Commun. 2014;5:4430.

12. Kang SK, Shin IS, Ko MS, Jo JY, Ra JC. Journey of mesenchymal stem cells for homing: strategies to enhance efficacy and safety of stem cell therapy. Stem Cells Int. 2012;2012(342968.

13. Eggenhofer E, Luk F, Dahlke MH, Hoogduijn MJ. The life and fate of mesenchymal stem cells. Front Immunol. 2014;5:148.

14. Gnecchi M, Zhang Z, Ni A, Dzau VJ. Paracrine mechanisms in adult stem cell signaling and therapy. Circ Res. 2008;103(11):1204-1219.

15. Hibaoui Y, Grad I, Letourneau A, Sailani MR, Dahoun S, Santoni FA, Gimelli S, et al. Modelling and rescuing neurodevelopmental defect of Down syndrome using induced pluripotent stem cells from monozygotic twins discordant for trisomy 21. EMBO Mol Med. 2014;6(2):259277.

16. Jiang J, Jing Y, Cost GJ, Chiang JC, Kolpa HJ, Cotton AM, Carone DM, et al. Translating dosage compensation to trisomy 21. Nature. 2013;500(7462):296-300.

17. Sloka JS, Stefanelli M. The mechanism of action of methylprednisolone in the treatment of multiple sclerosis. Mult Scler. 2005;11(4):425-432. 